\title{
DIFFÉRENTES SOURCES D'ERREURS DANS LES MESURES D'INTENSIMÉTRIE
}

\section{J. WASMER}

Société AKSUD, 11 allée des Imprimeurs, ZI Secteur A, F-06700 Saint Laurent du Var, France

\begin{abstract}
Résumé - Les principales causes d'erreurs qui affectent la précision des mesures d'intensité acoustique ont été exposées dans différentes publications: Nous en présentons ici une brève analyse, dont 1 e but est de pouvoir proposer des solutions pour la conception de sondes fiables ainsi que des moyens de vérification et d'étalonnage.

Abstract - The principal causes wich affect acoustical intensity measurements have been analysed in several publications. So here we only present a short analysis in order to propose new solutions in designing reliable sound intensity probes, as well as calibration and checking possibilities.
\end{abstract}

\section{INTRODUCTION}

Si l'être humain, doté seulement de ses deux oreilles comme capteurs et d'une partie de son cerveau comme analyseur, est capable de localiser en trois dimensions et en temps réel une source sonore et d'en déceler le déplacement de seulement deux degrés, il ne sait pas rêaliser un intensimètre comparable sans faire appel à au moins 4 mitarophonés. (I).

D'autre part, pour que les résultats d'une analyse d'intensimétrie soient valables, il faut impérativement en connaitre les limites. Or ces dernières sont liées aux différentes causes d'erreurs dans les mesures, mais aussi aux incertitudes d'étalonnage et aux dérives en fonction du temps et des conditions d'environnement.

\section{SOURCES D'ERREURS}

Une connaissance objective et suffisamment complète des sources d'erreurs est donc nécessaire. Nous examinerons principalement celles qui sont liées à la soncle et à l'étalonnage, mais nous ne considérerons pas celles qui sont dâes au traitement de signal, tout en restant conscients qu'elles sont à prendre en compte. 


\subsection{Approximation par différences finies}

Dans cette approximation, on admet que 1'intensité acoustique en un point donné, définie comme étant le produit de la pression et de la vitesse particulaireen ce point:

$$
I=\overline{p \cdot u} \quad \text { où }: u=-\frac{1}{\rho} \int \frac{\partial p}{\partial x} d t
$$

peut s'exprimer par:

soit:

$$
\begin{aligned}
& I=\frac{p_{A}+p_{B}}{2} x\left[-\frac{1}{p} \int \frac{p_{B}-p_{A}}{\Delta r} d t\right] \\
& I=-\frac{p_{A}+p_{B}}{2 p \Delta r} \int\left(p_{B}-p_{A}\right) d t
\end{aligned}
$$

où $\mathrm{p}_{\mathrm{A}}$ et $\mathrm{p}_{\mathrm{B}}$ sont les pressions en deux points $A$ et $\mathrm{B}$ situés symétriquement de part et d'autre du point donné:et séparés de $\Delta r: P$ représente la densité de 1 'air.

Ainsi, une sonde constituée de 2 microphones dont les centres acoustiques sont placés aux points A et B ne permettra un calcul valable de l'intensité que si l'on peut admettre:

$$
\frac{\partial p}{\delta r} \simeq \frac{p_{B}-p_{A}}{\Delta r} \quad \text { et } p \simeq \frac{p_{A}+p_{B}}{2}
$$

Or, dans la pratique, ces conditions sont satisfaites quand la longueur d'onde est grande devant la distance $\Delta r$ séparant 1 es 2 microphones. Ainsi, par exemple, pour une distance $\Delta r$ de $12 \mathrm{~mm}$, 1 'erreur sera inférieure à laB pour les fréquences inférieures à $5 \mathrm{kHz}$.

\subsection{Effets de 1a diffraction}

La diffraction se manifeste de la manière suivante 1orsqu'un microphone est placé dans un champ acoustique: la pression effective mesurée se compose de la pression qui existerait en son absence et de celle dâe à la diffraction causée par sa présence.

Les phénomènes de diffraction sont importants quand 1'obstacle causé par le microphone a des dimensions non négligeables devant la longueur d'onde.

Dans le cas d'une sonde d'intensimétrie comportant deux ou plusieurs microphones, 1 'étude des perturbations daes à la diffraction doit prendre en compte 1 'ensemble de la structure.

Des études de diffraction autour de sondes de formes simples, cylindriques. (2) et sphériques (3) ont été conduites et ont montré qu'il est possible de concevoir de telles sondes en tenant compte de corrections dans le traitement du signal. Toutefois, ces types de corrections ne sont applicables que si 1 'on peut négliger les effets de diffraction dás aux autres éléments de la structure de la sonde: support, poignée, etc...

Un autre effet des phénomènes de diffraction concerne la variation en fonction de la fréquence de la distance effective $\Delta r$ des centres acoustiques des deux microphones. Des études ont montré que 1 'on obtient de bons résultats sur une sonde unidimentionnelle cylinarique comportant 2 microphones disposés face à face et séparés par une cale d'espacement (4),: (5). 
La diffraction peut être aussi à l'origine de différence de sensibilité aux fréquences élevées des microphones entre 1 'incidence $0^{\circ}$ et $1^{\prime \prime}$ incidence $180^{\circ}$.

Enfin, la diffraction peut être à l'origine d'erreurs quadrantales qui ont pour conséquences que la sonde ne suit plus de manière satisfaisante la loi du cosinus aux fréquences élovéos, lorsqu'on fait varier 1 'angle d'incidence.

\subsection{Fonction de transfert des microphones}

Etant donné que le calcul de 1 'intensité acoustique implique la connaissance simultanée de la pression et de son gradient local, ce dernier étant lié directement au déphasage entre les deux voies microphoniques de mesure, il apparait clairement que le résultat peut être faussé s'il y a disparité entre la sensibilité en fonction de la fréquence des deux microphones, de même qu'entre leur réponse en phase en fonction de la fréquence.

\subsubsection{Réponse en fréquence}

Si la sensibilité des microphones diffère à la fréquence de $250 \mathrm{~Hz}$ choisie comme référence, l'écart peut être compensé au cours de 1 'étalonnage de 1 'ensemble sonde et analyseur.

Les différences de sensibilité en fonction de la fréquence sont fixées par le projet de norme CEI 1043 concernant les appareils de mesure d'intensimétrie. Il y a donc lieu de choisir des microphones convenablement appariés pour satisfaire ces exigences.

$2,3.2$. Réponse en phase

Aux fréquences basses, 1 e déphasage entre les deux points de mesure. représentés par les centres acoustiques des microphones este forcément faible en raison de la grande longueur d'onde.

Par exemple: 1 e déphasage d'une onde à $50 \mathrm{~Hz}$ qui se propage dans la direction des centres acoustiques des miarophones, séparés de $25 \mathrm{~mm}$, n'est que de 1,3 degré.

Il en résulte qu'une différence de réponse en phase à cette fréquence entre les deux microphones devient une source d'erreur dès qu'elle atteint 0,1 degré.

or la réponse en phase des microphones électrostatiques est conditionnée aux fréquences basses par le conduit d'égalisation de pression statique. Différentes solutions ont été appliquées pour réduire les écarts de réponse en phase des deux microphones aux basses fréquences:

-Appariement de la fréquence de coupure basse des deux microphones. -Abaissement de la fréquence de coupure des microphones en dessous de $1 \mathrm{~Hz}$.

- Egalisation de pression par un système multi-cavités (6).

Les deux premières solutions adoptées simultanément ou séparèment donnent des résultats peu satisfaisants à cause des variations dans 1 e temps et en fonction des conditions d'environnement. La troisième solution donne des résultats plus fiables.

Une autre solution consiste à traiter la sonde dans son ensemble. C'est celle qui a déjà été adoptée par AKSUD pour une sonde tridimentionnelle où le circuit d'égalisation de pression est en partie commun à $I$ 'ensemble des microphones $(7)$.

Aux fréquences élevées, la réponse en phase des microphones est déterminée par la fréquence de résonance et son amortissement. 
Ces effets ont fait l'objet d'études détaillées (8). Toutefois, nous retiendrons deux points essentiels:

10) Comme c'est à la fréquence de résonance que la phase varie le plus rapidement en fonction de la fréquence, il en résulte qu'une sonde avec 2 microphones ayant des fréquences de résonances différentes mais également amorties, présentera une différence de phase entre les deux voies d'autant plus grande que la sxituence sera proche de la moyenne entre les deux résonances. 20) Si les microphones ont la même fréquence de résonance mais des amortissements différents, c'est de part et d'autre que l'on rencontrera des écarts de phase.

Dans la pratique, on admet qu'il est préférable que la fréquence de résonance des microphones se situe largement au dessus de la gamme fréquentielle de mesure.

on notera également qu'une erreur de phase entre les deux voies microphoniques de $0,60 / \mathrm{kHz}$ n'aura pas d'incidence supérieure à 1 'exreur dâe à I'approximation par différences finies pour une sonde équipée de microphones espacés de plus de $6 \mathrm{~mm}$.

\subsection{Approximation du champ sonore}

Lorsqu'on assimile le champ sonore à une onde plane progressive, il peut en résulter une erreur appréciable en particulier pour les mesures d'intensité à proximité d'une source sonore où $1 a$ courbure du champ doit être prise en compte.

Toutefois, une analyse effectuée pour. une sonde de forme sphérique montre que $I$ 'erren est négligeable si. le rayon de courbure du champ sonore devient supérieur à 10 fois celui de la sonde (9)

\section{CONCEPTION D'UNE SONDE FIABLE}

AKSUD propose une sonde 1D de référence 9913 dont la conception nouvelle lui procure d'excellentes performances:

10) Le circuit d'égalisation des microphones passe par la structure de la sonde. Il est congu de sorte que l'atténuation résultante dépasse 6odB dans la gamme utile de fréquencé. Il en résulte que les microphones peuvent être considérés comme unipolaires pour 1 'étalonnage en phase de la sonde.

20) Les microphones proposés sont de type électrostatiques polarisés à fréquence de résonance élevée: $24 \mathrm{kHz}$ pour le modèle demi-pouce 3211 et $100 \mathrm{kHz}$ pour le modèle quart de pouce 3411 . La sonde ainsi équipée présente d'excentes performances de fiabilité en fonction du temps et des conditions d'environnement.

$\left.3^{\circ}\right)$ La sonde 9913 est équipée de grilles électrostatiques solidaires des microphones permettant l'étalonnage en phase de 1 'ensemble Sonde et Analyseur.

Il est à noter qu'il n'est pas nécessaire d'apparier les microphones si l'analyseur associé prend en compte l'étalonnage de la sonde pour le calcul de l'intensité.

\section{VERIFICATION ET ETALONNAGE}

Le calibreur d'intensimétrie 5699 permêt aussi bien l'étalonnage en phase et en amplitude des intensimètres équipés de la sonde 9913 , que les vérifications périodiques.

L'étalonnage en amplitude s'effectue en cavité par un signal de pression de $114 \mathrm{~dB}$ à $250 \mathrm{~Hz}$ ou $1 \mathrm{kHz}$.

L'étalonnage en phase utilise un générateur de bruit interne ou un Eénérateur externe. Il peut s'effectuer entre $20 \mathrm{~Hz}$ et $10 \mathrm{kliz}$ avec

les grilles électrostatiques de la sonde.
L'étalonnage en phase est aussi possible en cavité entre $20 \mathrm{~Hz}$ et 
$1 \mathrm{kHz}$. Cette possibilité permêt en particulier de déceler, en cours de vérification, des défauts tels que des micro-perforations des membranes qui ne sont pas manifestes pour l'étalonnage en grilles électrostatiques.

\section{CONCLUSION}

Nous avons pu montrer par cette approche qu'une bonne connaissance des principales sources d'erreurs dans les mesures d'intensimétrie constitue une aide importante dans la conception d'une sonde. D'autre part, si la fiabilité des microphones est suffisante, il n'est pas indispensable de les ajurier dans la mesure où 1'étalonnage de la sonde peut être pris en compte dans le calcul de $1^{\prime}$ intensité.

Enfin, si l'utilisateur d'une sonde 9913 peut effectuer des vérifications périodiques d'étalonnage avec le calibreur 5699, i1 pourra avoir une totale confiance en ses mesures.

(1) L'oreille principal organe de communication. Réveillez vous 22/1/90. Vo1 71, n'2. Watch Tower Bible and Tract Society of Pennsylvania.

(2) K. BELEFQIH et J.C. REBILLAT: Diffraction acoustique autour de sondes intensimétriques cylindriques..2e Congrès International sur 1 'Intensimétriè Acoustique. SENLIS 1985.

(3) 0. Coste et J.C. PATRAT: Diffraction autour d'une sphère appliquée à une sonde intensimétrique 3D. Ler Congrès Français d'Acoustique. LYON 1990.

(4) G. RASNUSSEN et M. BROCK: Acoustic intensity measurement probe. Congrès sur 1'Intensimétrie Acoustique. SENLIS 1981.

(5) P.V. BRUEL: Configurations de microphone pour sonde d'intensité acoustique. $2 e$ Congrés International sur 1 'Intensimétrie Acoustique. SENLIS 1985 .

(6) E. FREDERIKSEN: Caractéristiques de phase des microphones destinés aux sondes d'intensité. 2 e Congrés International sur I'Intensimétrie Acoustique. SENLIS 1985.

(7) J. WASMER: Sonde acoustique pour l'analyse d'intensimétrie en temps réel à 3 dimensions. ler Congrés Français d'acoustique. LYON 1990.

(8) T.M PHAN et J.C. PASCAL: La calibration en phase des sondes d'intens *métrie acoustiques par la méthode paramétrique. 2e Congrés International sur 1 'Intensimétrie Acoustique. SENLIS 1985.

(9) 0. COSTE et J.C. PATRAT: Scattering of a spherical wave applied to a $3 D$ sound intensity probe. Inter-Noise 1991. 